\title{
The fraction of quiescent massive galaxies in the early Universe
}

\author{
A. Fontana ${ }^{1}$, P. Santini ${ }^{1,2}$, A. Grazian ${ }^{1}$, L. Pentericci ${ }^{1}$, F. Fiore $^{1}$, M. Castellano ${ }^{1}$, E. Giallongo ${ }^{1}$, N. Menci ${ }^{1}$, \\ S. Salimbeni ${ }^{1,3}$, S. Cristiani ${ }^{4}$, M. Nonino ${ }^{4}$, and E. Vanzella ${ }^{4}$ \\ 1 INAF - Osservatorio Astronomico di Roma, via Frascati 33, 00040 Monteporzio (RM), Italy \\ e-mail: fontana@oa-roma.inaf.it \\ 2 Dipartimento di Fisica, Università di Roma "La Sapienza", P.le A. Moro 2, 00185 Roma, Italy \\ 3 Department of Astronomy, University of Massachusetts, 710 North Pleasant Street, Amherst, MA 01003, USA \\ ${ }^{4}$ INAF - Osservatorio Astronomico di Trieste, via G.B. Tiepolo 11, 34131 Trieste, Italy
}

Received 12 January 2009 / Accepted 21 January 2009

\section{ABSTRACT}

\begin{abstract}
Aims. We attempt to compile a complete, mass-selected sample of galaxies with low specific star-formation rates, and compare their properties with theoretical model predictions.

Methods. We use the $f(24 \mu \mathrm{m}) / f(K)$ flux ratio and the SED fitting to the $0.35-8.0 \mu \mathrm{m}$ spectral distribution, to select quiescent galaxies from $z \simeq 0.4$ to $z \simeq 4$ in the GOODS-MUSIC sample. Our observational selection can be translated into thresholds in specific star-formation rate $\dot{M} / M_{*}$, which can be compared with theoretical predictions.

Results. In the framework of the well-known global decline in quiescent galaxy fraction with redshift, we find that a non-negligible fraction $\simeq 15-20 \%$ of massive galaxies with low specific star-formation rate exists up to $z \simeq 4$, including a tail of "red and dead" galaxies with $\dot{M} / M_{*}<10^{-11} \mathrm{yr}^{-1}$. Theoretical models vary to a large extent in their predictions for the fraction of galaxies with low specific star-formation rates, but are unable to provide a global match to our data.
\end{abstract}

Key words. galaxies: formation - galaxies: evolution - galaxies: high-redshift

\section{Introduction}

Understanding the formation and evolution of early-type galaxies is a major goal of present-day cosmology, as well as a fundamental benchmark for "ab-initio" theoretical models of galaxy evolution.

According to several independent lines of evidence, the population of massive galaxies has undergone major evolution during the epoch corresponding to the redshift range 1.5-3, where the galaxy stellar mass function evolved significantly at high masses (Berta et al. 2007; Fontana et al. 2006, F06 in the following), massive galaxies settled onto the Hubble sequence (e.g., Abraham et al. 2007; and Franceschini et al. 2006), and the red sequence appears in high $z$ clusters (e.g., Zirm et al. 2008).

The nature of the physical processes responsible for this rapid rise remains unclear. A large number of massive $\left(\simeq 10^{11} M_{\odot}\right)$ actively star-forming galaxies is clearly in place at $z \simeq 2$ (Daddi et al. 2004; Papovich et al. 2007). Within this population, the more massive galaxies tend to be the more actively star-forming (Daddi et al. 2007b), at variance with trends measured in the local Universe.

At the same time, galaxies with low levels of star-formation rates at $z \simeq 1.5-2$ have been detected by imaging surveys based on color criteria (e.g., Daddi et al. 2004) or SED fitting (Grazian et al. 2007), and by spectroscopic observations of red galaxy samples (Cimatti et al. 2004; Saracco et al. 2005; Kriek et al. 2006). These results have motivated the inclusion of efficient methods for providing a rapid assembly of massive galaxies at high $z$ (such as starburst during interactions) as well as quenching of the SFR, most notably via AGN feedback (Menci et al. 2006; Bower et al. 2006; Hopkins et al. 2008).
Unfortunately, a detailed validation of the prediction of theoretical models has been hampered so far by the lack of a statistically well defined sample of early-type galaxies at high redshift, and by the difficulty in defining a common criterium to identify early-type galaxies in the data. It is difficult to isolate passively evolving galaxies from the wider population of intrinsically red galaxies at high redshift, which include also a (probably larger) fraction of dust-enshrouded star-forming galaxies. The two classes are indeed indistinguishable when selected by means of a single color criterium, such as the "ERO" classification $(R-K>4)$ (Daddi et al. 2000; McCarthy 2004) or the "DRG" one $(J-K>2$ ) (Franx et al. 2003; van Dokkum et al. 2003). The corresponding SEDs, however, are not degenerate and can be distinguished with more complex criteria, even when spectroscopy is not feasible. Some of these criteria adopt more colors, such as the $I-J / J-K$ method proposed by Pozzetti \& Mannucci (2000) and spectroscopically validated by Cimatti et al. (2003), or the $B z K$ method proposed by Daddi et al. (2004). Other methods rely on the spectral fitting of the overall SED, either by making use of the resulting rest-frame colors, as in the case of the $U-V / V-I$ criteria proposed by Wuyts et al. (2007), or directly using the output of the SED fitting process (Arnouts et al. 2007; Salimbeni et al. 2008; Grazian et al. 2007).

We used the method of using SED fitting output in our analysis of the GOODS-S data set, to disentangle the different contributors to the mass density at high redshift (Grazian et al. 2007) and to describe the evolution in the luminosity function of red galaxies up to $z \simeq 3$ (Salimbeni et al. 2008).

In this work, we use additional information available from observations in the mid-IR $24 \mu \mathrm{m}$ band, to allow a more careful selection of high redshift passively evolving galaxies, with the 
specific aim of comparing their number density with theoretical expectations.

We use a revised version of our GOODS-MUSIC sample (Grazian et al. 2006), a 15 band multicolor (U-to-24 $\mu \mathrm{m}$ ) catalog extracted from an area of 143.2 arcmin squared within the GOODS-South public survey. The key improvements compared to our previous work are a revised IRAC photometry and the addition of $24 \mu \mathrm{m}$ flux data for all objects in the catalog. Full details are given in a companion paper (Santini et al. 2009, S09 in the following). Another important difference is that we include all objects detected in the $4.5 \mu \mathrm{m}$ image, down to $m_{45}<23.5$. In the following, we adopt a mass-selected sample, obtained by applying a mass threshold at $M_{*} \geq 7 \times 10^{10} M_{\odot}$ to our photometric catalog based on a combined selection $K<23.5$ or $m_{45}<23.5$. This photometric sample is complete at this mass limit to $z \simeq 4$, also for dust-absorbed star-forming galaxies (see F06).

Observed and rest-frame magnitudes are in the AB system, and we adopt the $\Lambda$-CDM concordance model $\left(H_{0}=\right.$ $70 \mathrm{~km} \mathrm{~s}^{-1} \mathrm{Mpc}^{-1}, \Omega_{\mathrm{M}}=0.3$ and $\left.\Omega_{\Lambda}=0.7\right)$.

\section{Quiescent galaxies}

\subsection{The role of mid-IR observations}

In principle, the observed mid-IR flux is a powerful tool for distinguishing between the two classes of red galaxies at high redshift. Dust-absorbed star-forming galaxies are expected to be bright in the mid-IR, where most of the UV-light absorbed by dust is re-emitted. In contrast, passively evolving galaxies are expected to be far dimmer, since ordinary stars have low emission at IR wavelengths. To quantify this criterium, we use the ratio $f(24 \mu \mathrm{m}) / f(K)$ between the observed flux at $24 \mu \mathrm{m}$ and the $K$-band flux. We show the $f(24 \mu \mathrm{m}) / f(K)$ for all galaxies in our sample in Fig. 1, in four redshift bins. To show how this color can help us to differentiate between the galaxy types, we computed the same $f(24 \mu \mathrm{m}) / f(K)$ to $z \simeq 4$ for a set of local templates including early-type, spiral, and starburst galaxies (Polletta et al. 2007). All templates were $k$-corrected at the different redshifts. As shown in Fig. 1, the loci populated by the different galaxy types in the $U-V$ versus $f(24 \mu \mathrm{m}) / f(K)$ plane are separated well, allowing us to distinguish between actively star-forming galaxies and those with moderate-to-low star formation.

In Fig. 1, we show the observed $f(24 \mu \mathrm{m}) / f(K)$ for the complete $M_{*} \geq 7 \times 10^{10} M_{\odot}$ sample, both for individual galaxies as well as its general distribution. Upper limits were assumed to be equal to the $1 \sigma$ upper limits in the $24 \mu \mathrm{m}$ photometry. It is clearly shown in Fig. 1 that the $f(24 \mu \mathrm{m}) / f(K)$ ratio of star-forming galaxies increases with redshift, and that a significant population of starburst exists at $z>1$, with colors similar to those of local, rarer LIRGs. On the other hand, few bright galaxies at low and intermediate redshifts, with low star-formation rates are detected at low levels in the $24 \mu \mathrm{m}$ image.

The most striking result is that $f(24 \mu \mathrm{m}) / f(K)$ distribution is clearly bimodal, its minimum corresponding to a gap between the loci of active and passive galaxies. The observed minima in the distribution of the $f(24 \mu \mathrm{m}) / f(K)$ ratio occurs at $f(24 \mu \mathrm{m}) / f(K)=1,2,5$ and 6 in the four redshift bins adopted in Fig. 1. Two warnings are required about this bimodality. First, it is enhanced by the upper limits at faint $24 \mu \mathrm{m}$ levels, the true distribution probably being wider even at lower $f(24 \mu \mathrm{m}) / f(K)$. More importantly, the bimodality disappears if a deeper cata$\log$ is used (for instance, the $z \leq 26$ selected version of the GOODS-MUSIC catalog). The high mass-limit of our sample,

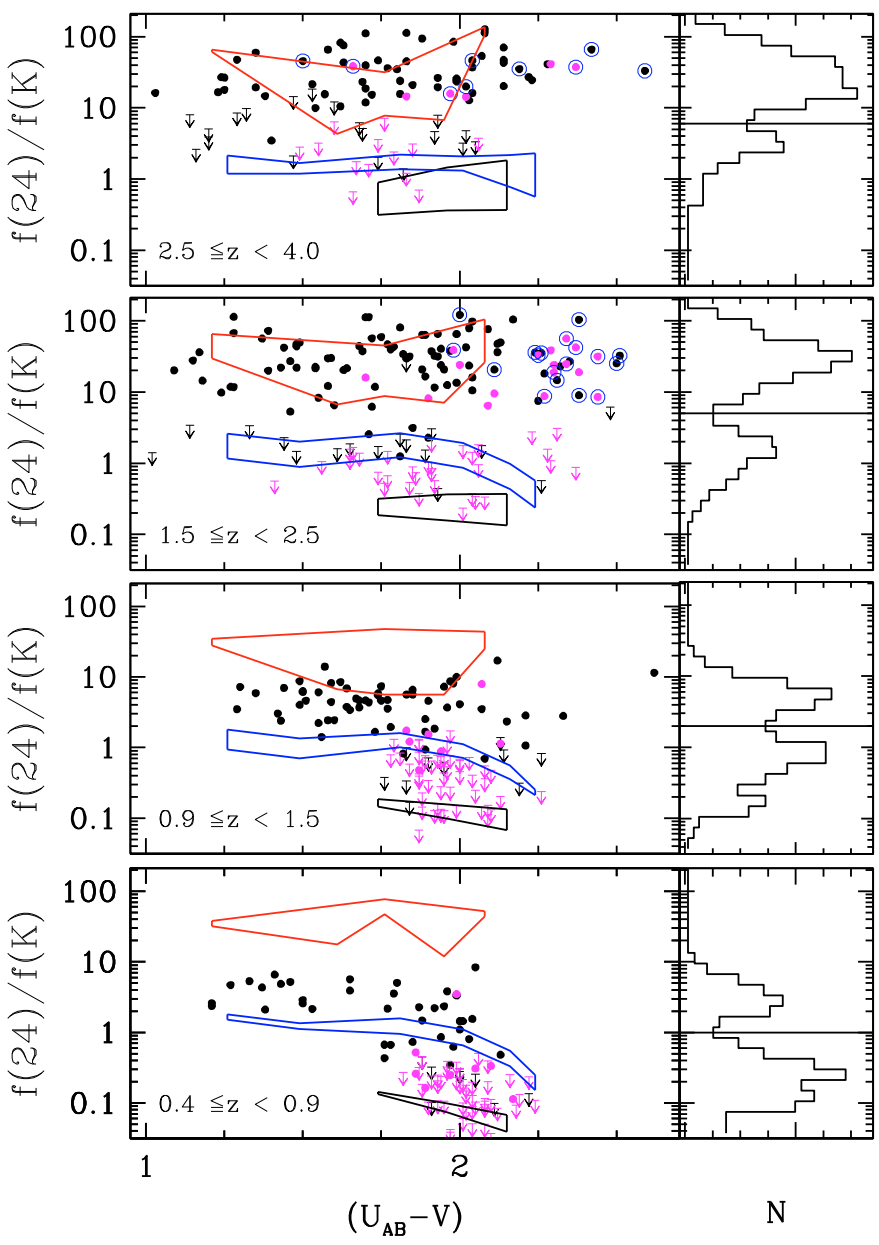

Fig. 1. Ratio of the observed fluxes at $24 \mu \mathrm{m}$ to that in the $K$ band as a function of the rest-frame $U-V$ color, in four redshift bins, for the $M_{*} \geq 7 \times 10^{10} M_{\odot}$ sample. Upper limits correspond to objects fainter than $20 \mu \mathrm{Jy}$ in the $24 \mu \mathrm{m}$ image. Galaxies with age $/ \tau>6$ (as discussed in Sect. 3) are shown in magenta. Large blue circles are the "obscured AGN" candidates of Fiore et al. (2008). Closed areas represent the range of $f(24 \mu \mathrm{m}) / f(K)$ obtained by redshifting a set of local samples (Polletta et al. 2007): from bottom to top, early-type galaxies (black line), spirals (blue line), starbursts (red line).

indeed, preferentially selects extremely red galaxies, as shown by their significantly red $U-V$ color, which are those for which the bimodality is clearly evident. This is a key feature of our approach, since the $f(24 \mu \mathrm{m}) / f(K)$ ratio provides a natural way of distinguishing between active and quiescent galaxies within the population of high-redshift, red galaxies.

\subsection{The selection on rest-frame quantities}

The criterium that we adopted to identify quiescent galaxies cannot be directly applied to the output of current theoretical models for galaxy formation and evolution, which typically do not predict the mid-IR flux. The comparison becomes more natural when we convert the data into the quantities immediately provided by these models, i.e., stellar mass or star-formation rates. We use the stellar masses $M_{*}$ provided by the SED fitting, of accuracy described at length in F06, and briefly in Sect. 3. To derive star-formation rates for galaxies detected at $24 \mu \mathrm{m}$, we apply the method of Papovich et al. (2007) in converting the $24 \mu \mathrm{m}+$ rest-frame UV luminosity into a total star-formation rate, using the Dale \& Helou (2002) models with a (lowering) correction 



Fig. 2. Ratio between the observed fluxes at $24 \mu \mathrm{m}$ and in the $K$ band as a function of the Specific Star-formation Rate $\left(\dot{M} / M_{*}\right)$, for the sample with $M_{*} \geq 7 \times 10^{10} M_{\odot}$. Galaxies with $\dot{M} / M_{*}<10^{-12} \mathrm{yr}^{-1}$ have been arbitrarily set to $\dot{M} / M_{*}=10^{-12} \mathrm{yr}^{-1}$. Upper limits refer to objects fainter than $20 \mu \mathrm{Jy}$ in the $24 \mu \mathrm{m}$ image. Large blue circles are the "obscured AGN" candidates of Fiore et al. (2008). The solid vertical line corresponds to the inverse of the age of the Universe at the centre of the redshift bin. The dashed vertical line shows the threshold on $\dot{M} / M_{*}$ adopted to classify "red and dead" galaxies. The horizontal solid line refers to the minimum in the observed distribution of the $f(24 \mu \mathrm{m}) / f(K)$ ratio in the same redshift range.

at high mid-IR fluxes. For galaxies below $20 \mu \mathrm{Jy}$ at $24 \mu \mathrm{m}$, we use the SFR derived from the SED fitting. As we show in S09 (see also Daddi et al. 2007b), these two SFR estimators agree relatively well, especially at low SFR levels.

In Fig. 2, we show the correlation between the $f(24 \mu \mathrm{m}) / f(K)$ ratio and $\dot{M} / M_{*}$ in our sample. For simplicity, we only plot the two redshift ranges that are more populated in our sample: the same trend holds at higher and lower redshifts. This correlation is largely expected given the relationship between $\dot{M}$ and the $24 \mu \mathrm{m}$ flux on the one hand, and between $M_{*}$ and the $K$ magnitude on the other. The key point, however, is that the correlation is so tight that it allows us to translate the (observational) criterium based on the $f(24 \mu \mathrm{m}) / f(K)$ ratio (horizontal line in Fig. 2) into a (model-oriented) cut to the estimated $\dot{M} / M_{*}$ (vertical line in Fig. 2). As shown in Fig. 2, the two samples in practice coincide.

Based on this evidence, we use the specific star-formation rate $\dot{M} / M_{*}$ to select quiescent galaxies, which allows direct comparison of the data with theoretical models. Given that $\dot{M} / M_{*}$ is dimensionally the inverse of a timescale, a natural threshold of $\dot{M} / M_{*}$ is the inverse of the age of the Universe at the corresponding redshift $\left(t_{U}(z)\right)^{-1}$. We define galaxies with $\dot{M} / M_{*}<\left(t_{U}(z)\right)^{-1}$ as "quiescent" in the following. Such a name is motivated by the fact that - by definition - these galaxies have experienced a major episode of star-formation prior to the observations ${ }^{1}$.

\footnotetext{
${ }^{1}$ Indeed, if $M_{*}=\langle\dot{M}\rangle_{\text {past }} \times t_{U}(z)$, where $\langle\dot{M}\rangle_{\text {past }}$ is the star-formation rate averaged over the whole age of the Universe at the corresponding $z$, the requirement $\dot{M} / M_{*}<\left(t_{U}(z)\right)^{-1}$ implies $\dot{M} \leq\langle\dot{M}\rangle_{\text {past }}$.
}

\section{3. "Red and dead" galaxies}

\subsection{Basic definitions}

Galaxies defined as "quiescent" by the criterium presented in the previous Sect. may still have a measurable amount of ongoing SFR (e.g., $\dot{M} \simeq 10 M_{\odot} /$ yr for a $M_{*} \simeq 10^{11} M_{\odot}$ galaxy). It is therefore interesting to estimate the fraction of galaxies with low or negligible levels of SFR. As we show in the following, the physical properties of these objects, that we label "red and dead", constitute a sterner test to theoretical models.

To select galaxies at high redshift on the basis of low starformation rates, we need to complement our $f(24 \mu \mathrm{m}) / f(K)$ ratio data with output from the fitting analysis of the optical-IR observed SED. The lack of detection in the $24 \mu \mathrm{m}$ image provides only an upper limit to the ongoing SFR (see for instance Fig. 2), and its exact level can be estimated from the SED fitting only. We applied the SED fitting technique following the recipe described in several papers (see Fontana et al. 2004; and F06 for details). The U-to- $8 \mu \mathrm{m}$ photometry was compared with a grid of models from the Bruzual \& Charlot 2003, BC03) spectral synthesis code, characterized by exponentially declining star-formation histories of timescale $\tau$ for a set of ages, metallicities, and dust extinctions. For comparison with our previous work, and most of the literature, we used the standard Salpeter IMF and the BC03 models. We verified however that adoption of the most recent version of the code incorporating the treatment of the post-AGB stars (Bruzual A. 2007) does not change our results significantly (see Salimbeni et al. 2009, for a preliminary analysis). In particular, only 1 (out of 144) objects classified as "red and dead" would not be classified as such with the new version.

To select "red and dead" galaxies, we use a threshold of $\dot{M} / M_{*}<10^{-11} \mathrm{yr}^{-1}$ (vertical dashed line in Fig. 2). To some extent, this threshold is arbitrary, the important point being that we adopt the same cut in theoretical models, to ensure proper comparison. However, we note that the same threshold was also used previously (Brinchmann et al. 2004) to separate active and quiescent galaxies. We also note that it corresponds to a threshold age $/ \tau>6$ between the fitted age and the starformation exponential timescale $\tau$. This follows from the fact that, assuming exponentially declining star-formation histories, the specific star-formation rate can be computed analytically to be $\dot{M} / M_{*}=\left(\tau\left(\mathrm{e}^{(\mathrm{age} / \tau)}-1\right)\right)^{-1}$, yielding $\dot{M} / M_{*} \simeq 10^{-11} \mathrm{yr}^{-1}$ for age $/ \tau \simeq 6$, for small values of $\tau$. This coincidence allows us to compare the pure SED fitting approach with the additional analysis provided by the $24 \mu \mathrm{m}$ data. A threshold to age $/ \tau$ was applied before (Arnouts et al. 2007; Salimbeni et al. 2008; Grazian et al. 2007) to broadly identify passively evolving galaxies, although with a less conservative value of age $/ \tau>4$. In Fig. 1, we plot the location in the $U-V$ vs. $f(24 \mu \mathrm{m}) / f(K)$ plane of all galaxies with age $/ \tau>6$ (magenta points). The classification scheme based purely on the optical-near IR SED fitting agrees well with the $f(24 \mu \mathrm{m}) / f(K)$ criterium, providing an important consistency check. The main exception are a number of "Compton thick AGN" candidates at $1.5<z<2.5$, selected as described in Fiore et al. (2008), where the $24 \mu \mathrm{m}$ emission was attributed to AGN activity. These objects are discussed further in Sect. 3.4.

We select "red and dead" galaxies by requiring that $\dot{M} / M_{*}<$ $10^{-11} \mathrm{yr}^{-1}$, not only that age $/ \tau>6$. This is because when estimating SFR, we assign the SED-derived values only to objects undetected in the mid-IR, i.e., those with $24 \mu \mathrm{m}$ flux below $20 \mu \mathrm{Jy}$. For this reason, the few objects fitted with age $/ \tau>6$ but 


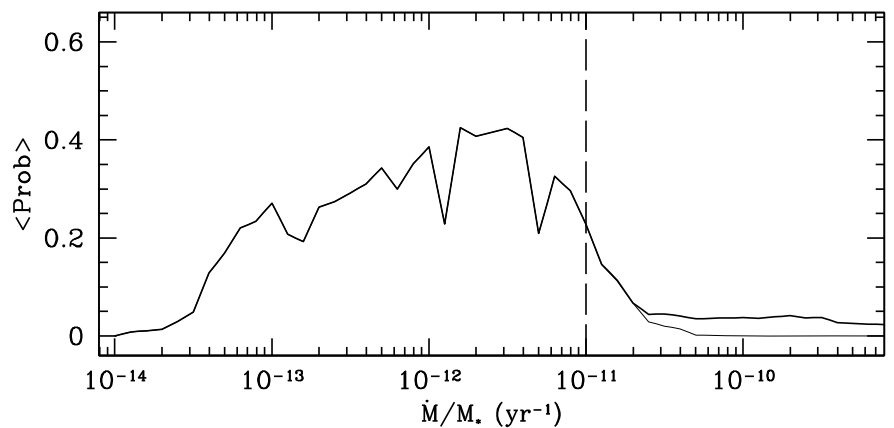

Fig. 3. Probability distribution of the $\dot{M} / M_{*}$ ratio in the sample of "red and dead" galaxies at $1.5 \leq z \leq 2.5$, averaged over the whole sample. The thick curve shows the probability distribution of the SED fitting to the 14 bands SED, from the $U$ band to the $8 \mu \mathrm{m}$ band. The thin curve shows the probability distribution removing models that over predict the SFR with respect to the upper limit provided by the constraint at $24 \mu \mathrm{m}$. The dashed vertical line shows the limit $\dot{M} / M_{*}<10^{-11} \mathrm{yr}^{-1}$ that we adopt to define "red and dead" galaxies.

with large $f(24 \mu \mathrm{m}) / f(K)$ are not included in our sample of "red and dead" galaxies. We now discuss the accuracy of this method for the most interesting subsample, i.e., "red and dead" galaxies at $z \geq 1.5$.

\section{2. "Red and dead" galaxies at $1.5 \leq z \leq 2.5$}

A sizeable fraction of the sample with $\dot{M} / M_{*}<10^{-11} \mathrm{yr}^{-1}$ have redshifts in the range $1.5 \leq z \leq 2.5$, where we detect 32 galaxies. The reliability of their $\dot{M} / M_{*}$ measure can be assessed using the error analysis already widely adopted in similar cases (Papovich et al. 2001, F06). Briefly, the full synthetic library used to determine the best-fit model spectrum is compared with the observed SED of each galaxy. For each spectral model (i.e., for each combination of the free parameters age, $\tau, Z$ and $E(B-V))$ the probability $P$ of the resulting $\chi^{2}$ is computed and retained, along with the associated $\dot{M}$ and $M_{*}$. The ensemble of $\dot{M} / M_{*}$ values corresponding to probabilities above a given minimum threshold defines the range of acceptable values for $\dot{M} / M_{*}$. Since most of our "red and dead" galaxies have only a photometric redshift, our analysis for these objects has been completed by allowing the redshift parameter to be free. In Fig. 3, we show the resulting distribution of the probability $P$, averaged over the entire sample of "red and dead" galaxies at $1.5 \leq z \leq 2.5$. The tail at large values of $\dot{M} / M_{*}$ is due to 2 objects with a nearly equal probability of being fitted accurately by a dusty starburst model solution. The correspondingly large values of $\dot{M}$ that would be required would easily overpredict the observed flux (which is an upper limit) at $24 \mu \mathrm{m}$. Taking into account this additional constraint, we display in Fig. 3 the distribution of $P$ after removing the models that overpredict the SFR with respect to the value provided by the upper limit in the emission at $24 \mu \mathrm{m}$. It is clear from Fig. 3 that the SED fitting for these galaxies is well constrained at levels of $\dot{M} / M_{*}<10^{-11} \mathrm{yr}^{-1}$, since the probability of having $\dot{M} / M_{*}$ has only a small tail above $10^{-11} \mathrm{yr}^{-1}$.

This result is unsurprising. Since the galaxies in our sample are by selection massive $\left(M_{*} \geq 7 \times 10^{10} M_{\odot}\right)$, the threshold $\dot{M} / M_{*}=10^{-11} \mathrm{yr}^{-1}$ is equivalent to small but measurable amounts of SFR, of approximately $1 M_{\odot} \mathrm{yr}^{-1}$. These levels correspond to detectable fluxes in the deep $B-z$ optical images of GOODS (the magnitude computed from a galaxy with $\simeq 1 M_{\odot} \mathrm{yr}^{-1}$, for small values of $E(B-V)$, is in the range 26-28 mag) and can then be measured by the full SED fitting.
We can also check the small subset of our "red and dead" sample with spectroscopic observations in this redshift range. Using both the K20 and the public GOODS data set, we found 6 objects that have a spectroscopic redshift. Four of these objects are classified as early spectral type, with no detectable [OII] emission line. Two other objects have both absorption features, typical of evolved systems, and a weak [OII] line. A weak [OII] line is not necessarily an indicator of ongoing starformation (Yan et al. 2006). Adopting the standard conversion of Kennicutt (1998), we derive star formation rates of the order of 2-3 $M_{\odot} \mathrm{yr}^{-1}$, consistent with the SED fitting estimates, confirming that these objects have $\dot{M} / M_{*} \lesssim 10^{-11} \mathrm{yr}^{-1}$. We conclude that our analysis is sufficiently robust at $1.5<z<2.5$, and allows to build a mass-selected sample of "red and dead" galaxies.

\section{3. "Red and dead" galaxies at $z>2.5$}

As we move to higher redshifts, $2.5 \leq z \leq 4$, our analysis becomes more uncertain. First, the constraints on the $24 \mu \mathrm{m}$ emission are weaker or non-existent, since this band is shifted outside the range dominated by dust emission. Objects also become even fainter and redder, and in general lack any spectroscopic confirmation. In this redshift range, we detect 12 "red and dead" candidates, 9 located at $2.5<z_{\text {phot }}<3$. We carefully examined the data for each object to assess the detection reliability. First, we verified that the photometry was not contaminated by nearby companions, and that the overall SED was smooth and not biased obviously by photometric fakes. All galaxies selected in this redshift range are very red with $z-B>4$, which is redder than typical EROs at lower redshifts. Their optical-IR SED is dominated by a break between the $K$ and the IRAC bands, and a change of slope that is a signature of an evolved galaxy population (star-forming, dusty objects exhibit a more featureless slope). The redshift probability distribution is broad, but typically implies that $z_{\text {phot }}>1.5-2$ with a relatively large spread $\Delta z_{\text {phot }} \simeq 0.5$.

Quantitatively, the error analysis described above indicated that 5 of the 9 objects at $2.5<z_{\text {phot }}<3$ are constrained to have $\dot{M} / M_{*}<10^{-11} \mathrm{yr}^{-1}$, even for such a broad redshift range. An example of these objects is shown in the upper panel of Fig. 4. The four other objects have a wider distribution of $\dot{M} / M$ (Fig. 4, middle panel), as well as redshift.

In a similar way, the physical properties and the nature of the three objects detected at $z>3$ are weakly constrained. They have a clear minimum in the $\chi^{2}$ distribution around $z_{\text {phot }} \simeq 3.5-4$, but have a tail to $z \simeq 2$. The distribution of acceptable $\dot{M} / M_{*}$ values also extends significantly beyond $10^{-11} \mathrm{yr}^{-1}$.

We conclude that at least $55 \%$ of our "red and dead" candidates at $2.5<z_{\text {phot }}<3$ are robust candidates, while the remaining $45 \%$ and the three candidates at $z>3$ are more uncertain. It would be natural to convert this finding into an upper limit to the true number density of "red and dead" galaxies. However, we note that a comparable number of galaxies are inferred to have $\dot{M} / M_{*}$ slightly above $10^{-11} \mathrm{yr}^{-1}$ and are not included in our sample, but have a range of acceptable model fits extending well below $\dot{M} / M_{*}=10^{-11} \mathrm{yr}^{-1}$. We conclude that at $z>2.5$ the estimate of $\dot{M} / M_{*}$ becomes unreliable, due mainly to the limitations in the depth of existing observations, and that the resulting statistical analysis of "red and dead" galaxies must be treated with caution. As we show, the statistical error (including both Poisson and cosmic-variance components) is so large for these small samples that a more rigorous treatment of these uncertainties is probably unnecessary. 


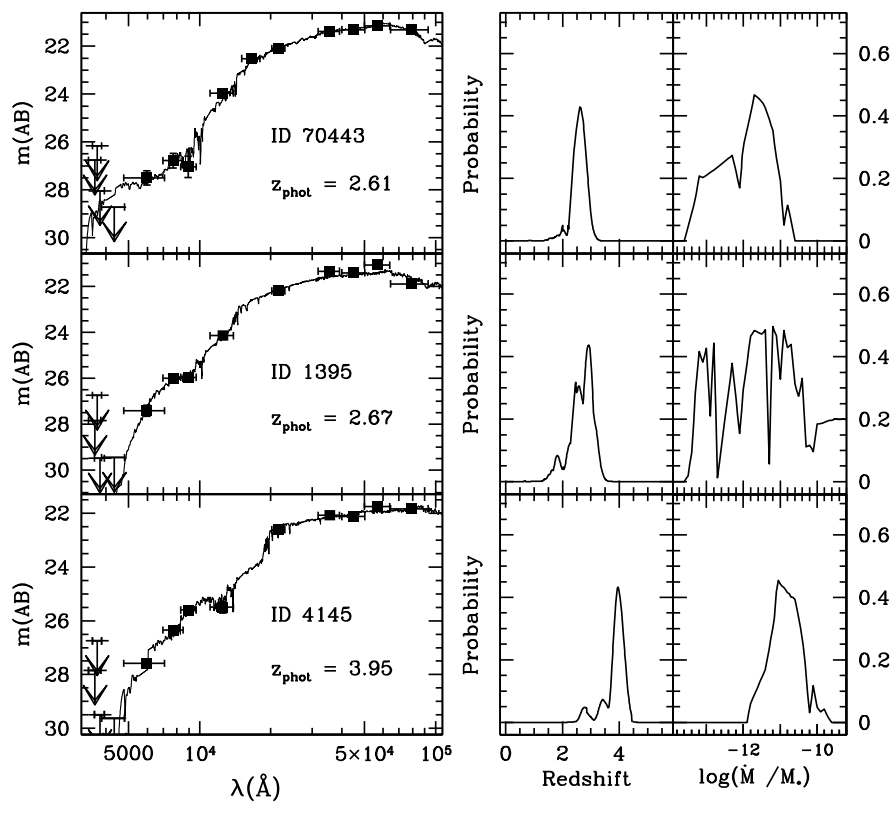

Fig. 4. Examples of "red and dead" galaxies at $z>2.5$. For each object, from left to right: the observed flux in the GOODS band and the bestfit SED; the redshift probability function; the $\dot{M} / M_{*}$ probability distribution. From top to bottom, the three galaxies shown represents three different categories: a robust candidate at $2.5<z<3$; a less secure candidate in the same redshift range; a typical example of the candidates at $z>3$.

\subsection{Hosts of Compton thick AGNs}

A potential source of uncertainties in many statistical analysis of high redshift galaxies is contamination by AGNs. As we describe in S09, we removed from our catalog both spectroscopically confirmed AGNs and X-ray sources with an optically dominant point-like source. We also identified galaxies that likely harbor Compton thick AGNs using the criterium defined by Fiore et al. (2008), which is based on the detection of a mid-IR excess in very red galaxies. This criterium is similar to the one adopted by Daddi et al. (2007a) but also includes objects that are much redder in the optical-IR range than the BzK-selected sample of Daddi et al. (2007a). A full discussion of the SED of these objects will be presented elsewhere: for the moment, we note that 9 of these "Compton thick AGN" candidates at $1.5<z<2.5$ are classified as "red and dead" galaxies, as shown in Fig. 1. Unfortunately, the nature of these objects remains elusive. Most of these objects are among the reddest in our sample, as indicated by their extreme rest-frame $U-V$. They are extremely faint or even undetected in the deep $z$-band ACS images, and are included in our sample only because of their detection in either $K$ or $4.5 \mu \mathrm{m}$ images. As a result, the probability distributions of their photometric redshifts are often broad, although they are typically constrained to be at $z_{\text {phot }}>2$. Only three (of nine) galaxies have the distribution of $\dot{M} / M_{*}$ constrained to be below $10^{-11} \mathrm{yr}^{-1}$. In the following, we do not rely on this classification, and we therefore compute the fraction of "red and dead" galaxies at $1.5<z<2.5$ in three different ways (labelled $a$ ), $b), c)$ in the caption of Fig. 5), to explore all possible cases. First (option $a$ )), we remove all "Compton thick AGN" candidates from our sample, irrespective of their SED classification. Alternatively (option $b$ )), we assume that none of them is a "red and dead" galaxy, which provides a lower limit to their fraction. Finally (option $c$ )), we include them in our statistical analysis, assigning their formal SED classification. As we show in the
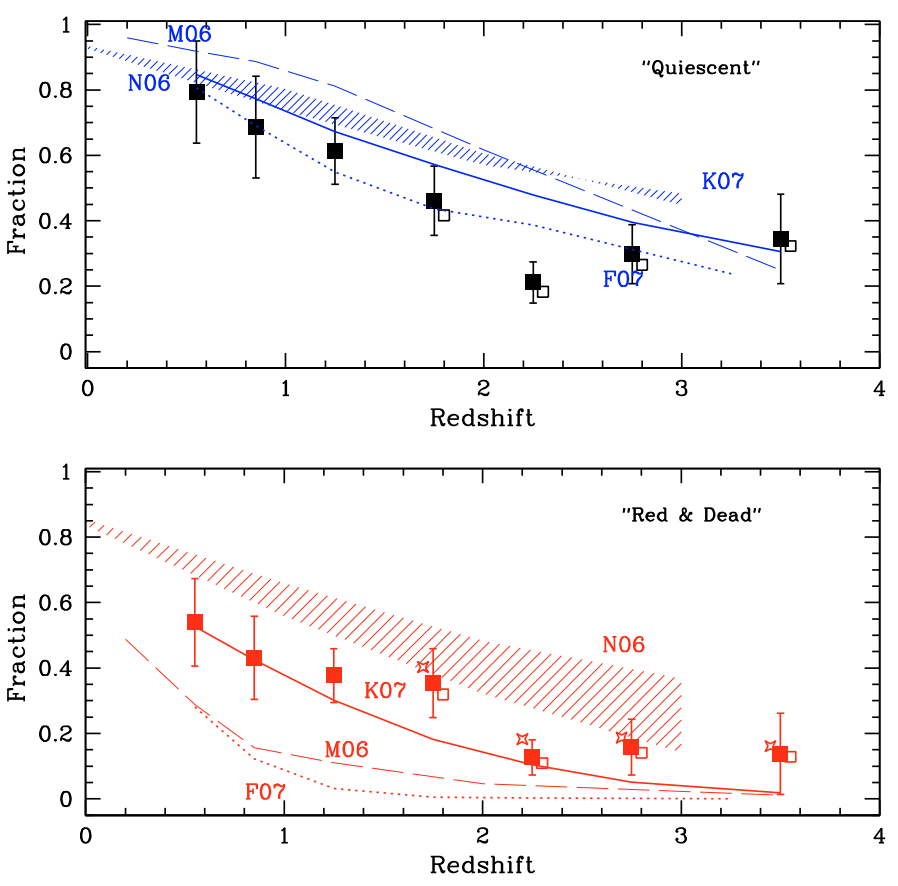

Fig. 5. Lower: fraction of "red and dead" galaxies (defined by $\dot{M} / M_{*}<$ $\left.10^{-11} \mathrm{yr}^{-1}\right)$ as a function of redshift, in the $M_{*} \geq 7 \times 10^{10} M_{\odot}$ massselected sample. Points represent the observed values. Filled, open and starred points (slightly offset for clarity) refer to the three different strategies $a, b, c$ discussed in the text to account for obscured AGNs. Error bars include Poisson and cosmic variance errors. Lines refer to the predictions of theoretical models, as described by the legend: Menci et al. (2006, M06), Fontanot et al. (2007, F07), Kitzbichler \& White (2007, K07), Nagamine et al. (2006, N06). Upper: fraction of "quiescent" galaxies as a function of redshift, defined by $\dot{M} / M_{*}<\left(t_{U}(z)\right)^{-1}$, in the same mass-selected sample. Points and lines as in the lower panel.

following section, the fraction computed with these different assumptions changes its value by $\simeq 20 \%$, still providing interesting constraints on theoretical models.

\section{Results and discussion}

We finally compute the fraction of "quiescent" and "red and dead" galaxies in different redshift ranges, following the definitions provided in the last two sections. These fractions are shown in Fig. 5 as a function of redshift. The different numbers of "red and dead" galaxies at $1.5<z<2.5$ reflect the different accounting methods of hosts of Compton thick AGNs. Error bars were computed by summing (in quadrature) the Poisson and cosmic variance error. The latter was computed by measuring the relative variance within 200 samples bootstrapped from the Millennium Simulation (Kitzbichler \& White 2007), using an area as large as GOODS-S and applying the same selection criteria.

We recall that these fractions are computed with respect to the total number of galaxies with $M_{*} \geq 7 \times 10^{10} M_{\odot}$ in our sample. Since we are interested in the ratio between galaxy classes, the impact of the exact choice of this threshold is measurable but not dramatic. We verified that by either increasing or decreasing the mass limit cut by a factor of 2 , the fraction of both "quiescent" and "red and dead" galaxies in different redshift ranges changes by about 0.1 , in the data (where applicable) as well as in the theoretical models. This factor of 2 variation may also be produced by adopting a different IMF, and is similar to the uncertainty in the stellar mass estimate. 
Our analysis confirms the cosmological decrease in the number density of massive early-type galaxies at high redshifts: quiescent galaxies dominate the population of massive galaxies at $z \ll 1$, and become progressively less common at higher $z$. However, we note that a significant fraction of galaxies with low levels of SFR is in place even at the highest redshifts sampled here $(z \simeq 3.5)$ with a fraction of about $10-15 \%$ at $z>2$.

By definition, "quiescent", massive galaxies assembled most of their stellar mass in previous epochs, implying that they experienced an active starburst phase or important merging process at higher redshifts. The large fraction of "quiescent" galaxies that we observe at $z \simeq 2$ implies that these processes must have been frequent at very high redshifts. The upper panel of Fig. 5 can also be interpreted in terms of "active" galaxies, i.e., the complementary galaxy fraction with $\dot{M} / M_{*} \geq\left(t_{U}(z)\right)^{-1}$. According to the duty-cycle argument presented in S09, these actively star forming galaxies experienced a major episode of star-formation, potentially building up a substantial fraction of their stellar mass in this episode. Their fraction increases with redshift, constituting more than $50 \%$ of the massive galaxy population at $z \geq 2$.

Finally, the sizeable number of "red and dead" galaxies is already in place at $z>1.5$, implying that the star-formation episodes must be quenched either by efficient feedback mechanism and/or by the stochastic nature of the hierarchical merging process.

It is interesting to determine whether theoretical models agree with these observational results. In Fig. 5, we plot the predictions of several models, applying the same criteria based on the $\dot{M} / M_{*}$ values. We consider purely semi-analytical models (Menci et al. 2006, M06; Fontanot et al. 2007, F07), a semianalytical rendition of the Millennium $N$-body dark matter simulation (Kitzbichler \& White 2007, K07), and purely hydrodynamical simulations (Nagamine et al. 2006, N06). The final are presented for three different timescales $\tau$ of the star-formation rate (ranging from $2 \times 10^{7} \mathrm{yrs}$ to $2 \times 10^{8} \mathrm{yrs}$ ), and represented with a shaded area. All these models agree in predicting a gradual decline with redshift in the fraction of galaxies with a low SFR.

As far as the "quiescent" fraction is concerned, we note that most models agree quantitatively in their predictions at all redshifts. There is a general tendency to slightly overpredict the fraction of "quiescent" galaxies, although the relatively large error bars of our sample prevent firm conclusions from being drawn. As we show in more detail in S09 (see also Daddi et al. 2007b), this is the result of an overall underestimate of the median SSFR of massive galaxies, which increases the number of mildly star-forming galaxies that we detect within our selection criteria $\dot{M} / M_{*}<\left(t_{U}(z)\right)^{-1}$. The F07 models provides a noticeable exception to this process. A main success of this model, indeed, is its capability of reproducing the Scuba counts and the high associated SFR (Fontanot et al. 2007): it is unsurprising that it also predicts a large fraction of active galaxies, and hence a smaller fraction of "quiescent" ones.

Conversely, the predicted fraction of "red and dead" galaxies varies significantly at all redshifts. This reveals that the predicted fraction of galaxies with very low levels of SFR is a particularly sensitive quantity, and provides a powerful way of highlighting the differences between the models. Some models (M06, F07) underpredict the fraction of "red and dead" galaxies at all redshifts, and in particular predict virtually no object at $z>2$, in contrast to what observed. The Millennium-based model agrees with the observed quantities, while the hydro model appears to overpredict them.
It is beyond the scope of the present paper to discuss the origins of these differences. They are difficult to ascertain, because of the complex interplay between all the physical processes involved in these models, the different physical process implemented - most notably those related to AGN feedback - and their different technical implementations. The failure of most models to reproduce simultaneously the fraction of "quiescent" and "red and dead" massive galaxies in the early Universe probably implies that the balance between the amount of cool gas and the star-formation efficiency on the one side, and the different feedback mechanisms on the other, is still poorly understood.

Acknowledgements. We are grateful to Mark Dickinson, Roberto Maiolino and Pierluigi Monaco for the useful discussions. We also thank K. Nagamine for providing the output of his models. We are also in debt with the two referees for useful and prompt suggestions, that improved the presentation of the work. This work is based on observations carried out with the Very Large Telescope at the ESO Paranal Observatory under Program ID LP168.A-0485 and ID 170.A-0788 and the ESO Science Archive under Program IDs 64.O-0643, 66.A-0572, 68.A0544, 164.O-0561, 163.N-0210, and 60.A-9120. The Millennium Simulation databases used in this paper and the web application providing online access to them were constructed as part of the activities of the German Astrophysical Virtual Observatory. We acknowledge financial contribution from contract ASI I/016/07/0 (COFIS).

\section{References}

Abraham, R. G., Nair, P., McCarthy, P. J., et al. 2007, ApJ, 669, 184 Arnouts, S., Walcher, C. J., Le Fèvre, O., et al. 2007, A\&A, 476, 137 Berta, S., Lonsdale, C. J., Polletta, M., et al. 2007, A\&A, 476, 151 Bower, R. G., Benson, A. J., Malbon, R., et al. 2006, MNRAS, 370, 645 Brinchmann, J., Charlot, S., White, S. D. M., et al. 2004, MNRAS, 351, 1151 Bruzual A., G. 2007, ArXiv Astrophysics e-prints

Bruzual, G., \& Charlot, S. 2003, Royal Astronomical Society, Monthly Notices, 344,1000

Cimatti, A., Daddi, E., Cassata, P., et al. 2003, A\&A, 412, L1

Cimatti, A., Daddi, E., Renzini, A., et al. 2004, Nature, 430, 184

Daddi, E., Cimatti, A., Pozzetti, L., et al. 2000, A\&A, 361, 535

Daddi, E., Cimatti, A., Renzini, A., et al. 2004, ApJ, 617, 746

Daddi, E., Alexander, D. M., Dickinson, M., et al. 2007a, ApJ, 670, 173

Daddi, E., Dickinson, M., Morrison, G., et al. 2007b, ApJ, 670, 156

Dale, D. A., \& Helou, G. 2002, ApJ, 576, 159

Fiore, F., Grazian, A., Santini, P., et al. 2008, ApJ, 672, 94

Fontana, A., Pozzetti, L., Donnarumma, I., et al. 2004, A\&A, 424, 23

Fontana, A., Salimbeni, S., Grazian, A., et al. 2006, A\&A, 459, 745

Fontanot, F., Monaco, P., Silva, L., \& Grazian, A. 2007, MNRAS, 382, 903

Franceschini, A., Rodighiero, G., Cassata, P., et al. 2006, A\&A, 453, 397

Franx, M., Labbé, I., Rudnick, G., et al. 2003, ApJ, 587, L79

Grazian, A., Fontana, A., De Santis, C., et al. 2006, A\&A, 449, 951

Grazian, A., Salimbeni, S., Pentericci, L., et al. 2007, A\&A, 465, 393

Hopkins, P. F., Cox, T. J., Kereš, D., \& Hernquist, L. 2008, ApJS, 175, 390

Kennicutt, Jr., R. C. 1998, ARA\&A, 36, 189

Kitzbichler, M. G., \& White, S. D. M. 2007, MNRAS, 376, 2

Kriek, M., van Dokkum, P. G., Franx, M., et al. 2006, ApJ, 649, L71

McCarthy, P. J. 2004, ARA\&A, 42, 477

Menci, N., Fontana, A., Giallongo, E., Grazian, A., \& Salimbeni, S. 2006, ApJ, 647,753

Nagamine, K., Ostriker, J. P., Fukugita, M., \& Cen, R. 2006, ApJ, 653, 881

Papovich, C., Dickinson, M., \& Ferguson, H. C. 2001, ApJ, 559, 620

Papovich, C., Rudnick, G., Le Floc'h, E., et al. 2007, ApJ, 668, 45

Polletta, M., Tajer, M., Maraschi, L., et al. 2007, ApJ, 663, 81

Pozzetti, L., \& Mannucci, F. 2000, MNRAS, 317, L17

Salimbeni, S., Giallongo, E., Menci, N., et al. 2008, A\&A, 477, 763

Santini, P., Fontana, A., Grazian, A., et al. 2009, A\&A, submitted

Saracco, P., Longhetti, M., Severgnini, P., et al. 2005, MNRAS, 357, L40

van Dokkum, P. G., Förster Schreiber, N. M., Franx, M., et al. 2003, ApJ, 587, L83

Wuyts, S., Labbé, I., Franx, M., et al. 2007, ApJ, 655, 51

Yan, R., Newman, J. A., Faber, S. M., et al. 2006, ApJ, 648, 281

Zirm, A. W., Stanford, S. A., Postman, M., et al. 2008, ApJ, 680, 224 